\title{
Commingling and Segregation Analyses: Comparison of Results From a Simulation Study of a Quantitative Trait
}

\author{
Jennifer M. Kwon, Michael Boehnke, Trudy L. Burns, and Patricia P. Moll \\ Departments of Epidemiology (J.M.K., P.P.M.), Biostatistics (M.B.), and Human \\ Genetics (P.P.M.), University of Michigan, Ann Arbor; Department of Preventive \\ Medicine and Environmental Health, University of lowa, lowa City (T.L.B.)
}

Commingling analysis is commonly used to provide preliminary evidence for a single genetic locus with a major effect on the quantitative trait of interest. In this paper, the effectiveness of commingling analysis as a screening technique to identify samples for segregation analysis is assessed by applying both commingling and segregation analyses to samples of simulated pedigree data in which a major locus is segregating in the presence of polygenes and an individual-specific environmental effect. Under the circumstances simulated here, there is evidence for a single locus from segregation analysis but not from commingling analysis in at least $20 \%$ of the samples. No more than $2 \%$ of the samples provided evidence for commingling but not for segregation of a single locus. Comparisons of the samples that give evidence for both commingling and segregation, evidence for one but not the other, and no evidence for either show that evidence for commingling depends on the distributional characteristics of the trait in the sample, while support for the single locus from segregation analysis depends on both the distributional characteristics as well as the transmission of the rarer allele from parents to offspring. Since lack of commingling does not rule out the existence of a single locus in the realistic situations considered here, commingling analysis has limited usefulness as a screening technique for the presence for a single locus. In contrast, evidence for commingling does suggest the possibility that a single locus has a major effect on the trait and commingling analysis can provide guidance in the choice of initial parameter estimates for segregation analysis.

Key words: screening, quantitative traits, pedigree analysis, admixture

Received for publication August 4, 1989; revision accepted November 2, 1989.

Address reprint requests to Michael Boehnke, Ph.D., Department of Biostatistics, School of Public Health, University of Michigan, 109 South Observatory, Ann Arbor, MI 48109-2029.

(C) 1990 Wiley-Liss, Inc. 


\section{INTRODUCTION}

When segregation at a single genetic locus has a major effect on the observed variation in a quantitative trait, each genotype at that locus has a particular associated phenotypic distribution, and the overall population distribution results from the commingling of these genotype-specific distributions. In general, commingling in the observed distribution of a quantitative trait may be caused by variability in a single factor, genetic or not [Murphy, 1964; Morton et al., 1977]. Evidence for a mixture of distributions is consistent with the hypothesis of a single genetic locus with a major effect on the trait of interest [Elston et al., 1975]. For this reason, commingling analysis is commonly used in samples of unrelated individuals [Morton et al., 1977; Rice et al., 1982; Turner et al., 1985; Richelson et al., 1986; Price et al., 1989] or related individuals [Bucher et al., 1982; McGue et al., 1983; Friedlander et al., 1984; Sharma et al., 1984; Bogardus et al., 1988; Turner et al., 1989] to test the hypothesis of a mixture of distributions.

Some studies of related individuals have considered the results from both commingling analysis and segregation analysis to interpret whether a single locus hypothesis is consistent with the observed data [Meyers et al., 1982; Dorus et al., 1983; Laskarzewski et al., 1984; Rice et al., 1984; Boerwinkle et al., 1986; Price et al., 1988; Moll et al., 1989; Olson et al., 1989]. In addition to providing preliminary evidence for a single factor with a major effect, commingling analysis can also provide guidance in the choice of initial parameter estimates for segregation analysis of family data [Elston et al., 1975].

In the present study, the effectiveness of commingling analysis as a screening technique to identify samples for segregation analysis is assessed by applying both commingling and segregation analyses to samples of simulated pedigree data in which a single locus is segregating in the presence of polygenes and individual specific environmental effects. Under the circumstances simulated here, there is evidence for a single locus from segregation analysis but not from commingling analysis in at least $20 \%$ of the samples. This finding suggests that the power of commingling analysis can be low relative to that of segregation analysis and that failure to find evidence for commingling should not preclude segregation analysis.

\section{MATERIALS AND METHODS Model and Simulation}

The quantitative data for this comparison study were generated to be representative of a sample of pedigrees each ascertained through a single proband whose phenotypic value exceeded the 95th percentile of a theoretical population distribution. The pedigree data were simulated under two mixed genetic models [Elston and Stewart, 1971; Morton and MacLean, 1974]; the distribution of the quantitative trait represents the summed effects of a major locus with two alleles, additive polygenes, and individualspecific environmental factors. Parameter values were chosen to simulate a trait that 1) showed dominance at the major locus, 2) had considerable overlap of the component distributions, 3) was unimodal in the general population, and 4) had a substantial polygenic component; such characteristics have been reported for several traits that are risk factors for common diseases [Sing et al., 1988]. 
We considered two different mixed genetic models. For Model I, the frequency of the dominant major locus allele $A$ was set at $q=0.012579$, so that $2.5 \%$ of the general population possessed genotype Aa or AA. The genotypic means were $\mu_{\mathrm{aa}}=$ 100 and $\mu_{\mathrm{Aa}}=\mu_{\mathrm{AA}}=117.28$. The within-distribution standard deviation $\sigma$ was set at 9.5 . This resulted in major locus genotype means separated by 1.75 phenotypic standard deviations. The within-distribution variance was divided evenly between the effects of additive polygenes and individual-specific environment. The composite population for Model I is shown in Figure 1. Model II was identical to Model I except the genotype means were $\mu_{\mathrm{aa}}=100$ and $\mu_{\mathrm{Aa}}=\mu_{\mathrm{AA}}=120$ so that the major locus genotype means were separated by 2.00 phenotypic standard deviations, rather than 1.75. Model II has previously been described in simulation studies by Burns [1982], Burns et al. [1984], and Boehnke et al. [1988].

Data were generated for the nine-person pedigree illustrated in Figure 2. This pedigree configuration has been shown in previous studies to provide the basis for an efficient study design for complex segregation analysis [Burns, 1982; Burns et al., 1984, Boehnke et al., 1988], and represents a compromise between small nuclear families and large extended pedigrees. Trait values for the pedigree members were simulated in the manner described by Boehnke et al. [1988]. If the trait value of the potential proband (designated by the arrow in Fig. 2) was in the upper 5\% of the trait distribution for the population, the proband was included in the sample and the pedigree was ascertained. Sampling continued until a total sample size of 50 pedigrees including $N$ $=450$ individuals was achieved. Five hundred replicate samples (each with $\mathrm{N}=450$ ) were generated using each of the two mixed models.

\section{Commingling Analysis}

The simulated data for each replicate sample were evaluated for the presence of a mixture of normal distributions. The pedigree members were treated as unrelated individuals for these analyses, and probands were excluded as a partial correction for ascer-

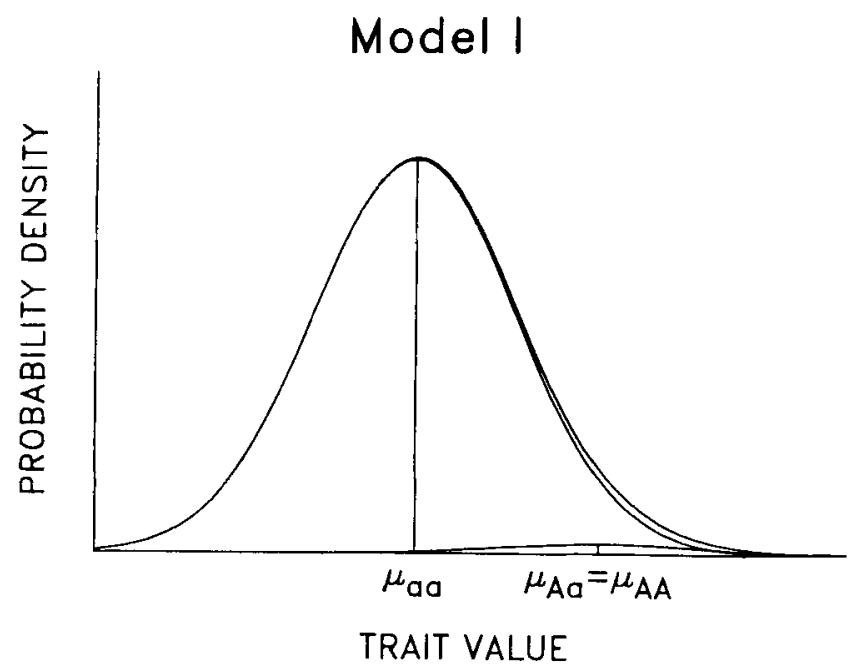

Fig. 1. The population distribution of the simulated phenotypes for Model $1, \mu_{\mathrm{aa}}=100, \mu_{\mathrm{Aa}}=\mu_{\mathrm{AA}}=$ 117.28 . 


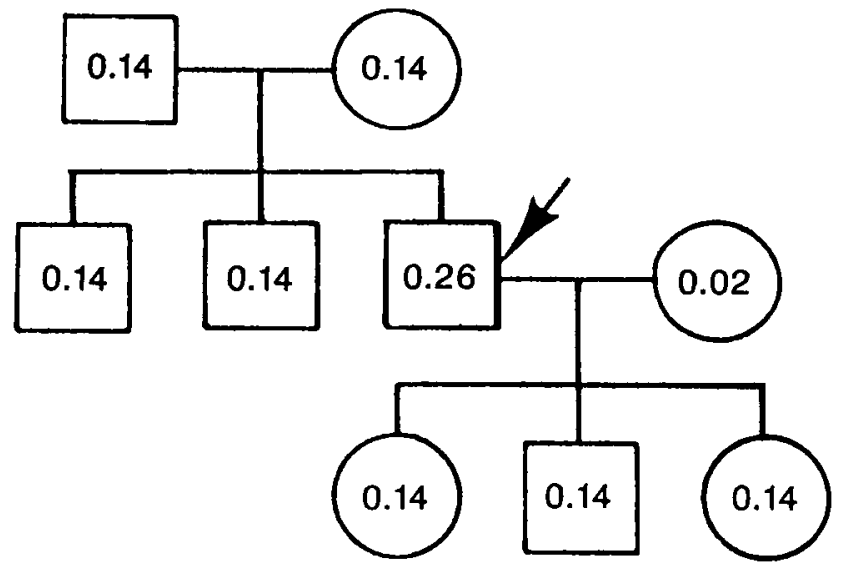

Fig. 2. Configuration of the nine-member pedigree used in the simulations. The potential proband is designated by the arrow. Numbers within the squares and circles represent the estimated probabilities for Model I that the corresponding individuals carry the major locus allele A as either a heterozygote or homozygote. Estimated probabilities are based on simulations of 20,000 replicate pedigrees.

tainment. Maximum likelihood methods suggested by Day [1969] were used to evaluate whether one or two normal distributions provided the better explanation for the data. Maximum likelihood estimates were obtained by using an EM algorithm [MacLachlan and Basford, 1988] with the implementation procedure suggested by Ott [1979]. The maximum log-likelihood for two component normal distributions with equal variances was compared to the maximum log-likelihood for a single normal distribution. Twice the difference between the log-likelihoods was assumed to be distributed approximately as chi squared with 2 degrees of freedom (df). A sample was classified as showing evidence of a mixture of two distributions if twice the difference between the loglikelihoods was greater then 4.605, corresponding to a significance level of $\alpha=0.10$.

\section{Segregation Analysis}

Log-likelihoods for the mixed genetic and polygenic models were computed for each replicate sample by using the Pedigree Analysis Package (PAP) [Hasstedt and Cartwright, 1981], which employs an approximation to the exact log-likelihood of a mixed model [Hasstedt, 1982]. Log-likelihoods were maximized by using a quasiNewton method [Lalouel, 1979]. Since ascertainment was necessarily single, we corrected for ascertainment by conditioning on the quantitative trait value of the proband in each pedigree [Hopper and Mathews, 1982; Boehnke and Lange, 1984; Young et al., 1988].

Twice the difference between the log-likelihoods of the mixed and polygenic models was assumed to be distributed approximately as chi squared with $2 \mathrm{df}$. A sample was classified as showing evidence of major locus segregation if twice the difference between the log-likelihoods was greater than 5.99, corresponding to a significance level of $\alpha=0.05$. The different significance levels chosen for commingling analysis ( $\alpha$ $=0.10)$ and segregation analysis $(\alpha=0.05)$ are consistent with the view of commingling analysis as a preliminary, screening technique and segregation analysis as a more definitive, confirmatory method. 


\section{Statistical Analysis of the Simulation Results}

Based on the outcome of the commingling and segregation analyses, the 500 replicate data sets were classified into four groups: 1) those that showed evidence both for a mixture of two distributions by commingling analysis and for major locus segregation by segregation analysis, 2) those that showed evidence for major locus segregation but not for commingling, 3) those that showed evidence for commingling but not for major locus segregation, and 4) those that showed evidence neither for commingling nor for major locus segregation. Sample characteristics for these four groups were then computed for the total sample including probands and then for all individuals except the probands. These included two major locus characteristics: the proportion of carriers of the dominant major locus allele and the mean number of parent/offspring pairs per pedigree both of whom carry the dominant allele; and the first four moments of the sample trait distribution: mean, variance, skewness, and kurtosis. Characteristics of the four groups were compared by using the analysis of variance. Multiple linear regression analysis was used to determine which characteristics were the best predictors of the results of the commingling and segregation analyses. Such an approach could not be taken by an investigator to infer the presence or absence of a major gene, but did allow us to identify those characteristics of the replicate samples that were important determinants of the results of the commingling and segregation analyses.

\section{RESULTS}

Table I cross classifies the 500 samples simulated under each of the two models by the significant or nonsignificant results for commingling and segregation analyses.

TABLE I. Cross Classification of the Results of the Commingling and Segregation Analyses*

\begin{tabular}{lccc} 
& \multicolumn{3}{c}{ Segregation analysis, No. (\%) } \\
\cline { 2 - 4 } Commingling analysis & $P \leqslant 0.05$ & $P>0.05$ & Total \\
\hline $\begin{array}{l}\text { Model I } \\
P \leqslant 0.10\end{array}$ & 206 & 10 & 216 \\
& $(41.2)$ & $(2.0)$ & $(43.2)$ \\
$P>0.10$ & 168 & 116 & 284 \\
& $(33.6)$ & $(23.2)$ & $(56.8)$ \\
Total & 374 & 126 & \\
& $(74.8)$ & $(25.2)$ & 388 \\
Model II & & 3 & $(77.6)$ \\
$P \leqslant 0.10$ & 385 & $(0.6)$ & 112 \\
& $(77.0)$ & 5 & $(22.4)$ \\
$P>0.10$ & 107 & $(1.0)$ & \\
Total & $(21.4)$ & 8 & $(1.6)$ \\
\hline
\end{tabular}

*Model I: Under this model, pedigrees were simulated from a population with means 1.75 phenotypic standard deviations apart. Model II: Under this model, pedigrees were simulated from a population with means 2.00 phenotypic standard deviations apart. 
If evidence for major locus segregation is regarded as "truth," use of commingling analysis as a screening method to detect major locus segregation resulted in a proportion of false negatives of 45\% (168/374) for Model I and 22\% (107/492) for Model II. Thus, using commingling analysis as a screening method would frequently prevent carrying out a segregation analysis that would provide significant evidence for major locus segregation under the circumstances simulated here, despite the more liberal significance level used for commingling analysis $(\alpha=0.10)$ than for segregation analysis $(\alpha=0.05)$.

Table II presents major locus characteristics and sample moments for the Model I samples divided into four groups according to whether there was significant evidence for commingling and/or for major locus segregation. While analysis of variance provided evidence ( $\alpha=0.05$ ) for differences between the four groups for all characteristics listed with the exception of kurtosis with probands included, actual differences between the groups in terms of standard deviation units were for the most part small. The groups differed primarily in skewness, which showed differences between groups of up to 1.9 standard deviations, and proportion of carriers of the dominant allele and mean number of parent-offspring transmissions of the dominant allele per family, which showed differences between groups of up to 1.0 standard deviation. All three of these measures appeared to have important effects on evidence for major locus segregation, while only skewness appeared to strongly impact evidence for commingling. Since some of the sample characteristics, notably skewness and kurtosis, were not normally

TABLE II. Characteristics of the Groups of Samples Classified by the Results of Commingling and Segregation Analyses, Model I

\begin{tabular}{|c|c|c|c|c|c|c|c|}
\hline \multirow[b]{4}{*}{ Statistic } & \multirow[b]{4}{*}{$\begin{array}{l}\text { Probands } \\
\text { included? }\end{array}$} & \multicolumn{4}{|c|}{ Segregation } & \multirow[b]{4}{*}{$\begin{array}{c}\text { Standard } \\
\text { deviation }^{a}\end{array}$} & \multirow[b]{4}{*}{$P$ value } \\
\hline & & \multicolumn{2}{|c|}{$P \leqslant 0.05$} & \multicolumn{2}{|c|}{$P>0.05$} & & \\
\hline & & \multicolumn{2}{|c|}{ Commingling } & \multicolumn{2}{|c|}{ Commingling } & & \\
\hline & & $P \leqslant 0.10$ & $P>0.10$ & $P \leqslant 0.10$ & $P>0.10$ & & \\
\hline \multirow{2}{*}{$\begin{array}{l}\text { Proportion } \\
\text { of carriers }\end{array}$} & Yes & 0.145 & 0.148 & 0.117 & 0.130 & 0.032 & $<0.0001$ \\
\hline & No & 0.116 & 0.118 & 0.094 & 0.103 & 0.026 & $<0.0001$ \\
\hline \multirow{2}{*}{$\begin{array}{l}\text { Mean no. of } \\
\text { transmissions } \\
\text { per pedigree }\end{array}$} & Yes & 1.017 & 1.042 & 0.816 & 0.911 & 0.236 & $<0.0001$ \\
\hline & No & 0.341 & 0.346 & 0.306 & 0.308 & 0.095 & $<0.005$ \\
\hline \multirow[t]{2}{*}{ Sample mean } & Yes & 107.8 & 107.7 & 107.7 & 107.6 & 0.638 & $<0.02$ \\
\hline & No & 106.1 & 106.0 & 105.9 & 105.8 & 0.693 & $<0.02$ \\
\hline \multirow{2}{*}{$\begin{array}{l}\text { Sample } \\
\quad \text { variance }\end{array}$} & Yes & 130.2 & 130.4 & 127.7 & 125.1 & 9.133 & $<0.0001$ \\
\hline & No & 116.5 & 116.7 & 113.4 & 110.7 & 9.642 & $<0.0001$ \\
\hline \multirow{2}{*}{$\begin{array}{l}\text { Sample } \\
\text { skewness }\end{array}$} & Yes & 0.150 & 0.025 & 0.115 & -0.022 & 0.103 & $<0.0001$ \\
\hline & No & 0.315 & 0.151 & 0.268 & 0.073 & 0.128 & $<0.0001$ \\
\hline Sample & Yes & 2.733 & 2.689 & 2.750 & 2.720 & 0.197 & $>0.15$ \\
\hline kurtosis & No & 3.210 & 3.042 & 3.237 & 3.056 & 0.276 & $<0.0001$ \\
\hline No. of samples & & 206 & 168 & 10 & 116 & & \\
\hline
\end{tabular}

${ }^{\text {aT }}$ The standard deviations reported are of the characteristics in the entire set of 500 replicate samples of 450 individuals each (if the probands are included) or 400 individuals each (if the probands are excluded). 
distributed and had unequal variances in the four groups, each sample characteristic was transformed to normality by using inverse normal scores [Daniel and Wood, 1980]. Analysis of variance on the normalized sample characteristics resulted in $P$ values nearly identical to those reported in Table II.

These findings are reinforced by the results of linear regression analysis of the likelihood ratio statistics for commingling and segregation from the 500 replicate simulations against the various characteristics of the samples for Model I. Likelihood ratio statistics were transformed to approximate normality by using a square root transformation prior to regression analysis. Skewness (excluding the probands) explained $86 \%$ of the variability in the transformed commingling likelihood ratio statistic; no other sample characteristic provided significant additional information. Residuals from the regression analysis appeared roughly normal and of constant variance. For segregation analysis, skewness (excluding the probands) explained $39 \%$ of the variability in the transformed likelihood ratio statistic; adding either the proportion of carriers of the dominant allele or the mean number of transmission per family of the dominant allele significantly improved the proportion of variability explained to $44 \%$. Residuals from the regression analysis appeared roughly normal and of constant variance. It is worth noting that as a predictor of the transformed likelihood ratio statistic for major locus segregation, skewness explained the same proportion of variability as could be explained by the transformed likelihood ratio statistic for commingling, that is, $39 \%$. The fact that skewness alone or skewness and mean number of transmissions of the dominant allele alone could not be significantly improved upon as explanations of the transformed likelihood ratio statistics was not due to collinearity among the major locus characteristics and sample moments. Among these predictors, only the proportion of carriers and the mean number of transmissions per pedigree were strongly correlated.

Results for Model II were qualitatively similar to those for Model I (data not shown), though the very small number of samples failing to provide evidence for major locus segregation makes their interpretation less clear cut.

We examined the distribution of the parameter estimates from commingling analysis to evaluate their usefulness as start values for subsequent segregation analysis. Because the distributions of several of the parameter estimates were non-normal, we report median values (Table III). For both models, the two genotype-specific means and particularly the dominant allele frequency were generally overestimated, probably due to the non-random sampling through the upper tail of the population distribution. In contrast, the within-distribution standard deviation was estimated quite accurately.

TABLE III. Median Parameter Estimates From the Commingling Analyses*

\begin{tabular}{llcccc} 
& & & & \multicolumn{2}{c}{ Model II } \\
\cline { 3 - 5 } Parameter & True value & & $\begin{array}{c}\text { Median } \\
\text { estimated value }\end{array}$ & True value & $\begin{array}{c}\text { Median } \\
\text { estimated value }\end{array}$ \\
\hline $\mathrm{q}$ & 0.012579 & 0.148 & 0.012579 & 0.155 \\
$\mu_{\mathrm{aa}}$ & 100.00 & 103.89 & 100.00 & 103.62 \\
$\mu_{\mathrm{A}-}$ & 117.28 & 118.61 & 120.00 & 121.47 \\
$\sigma$ & 9.50 & 9.35 & 9.50 & 9.45 \\
\hline
\end{tabular}

*Median parameter estimates based on commingling analyses on 500 samples of 400 individuals each (since probands were excluded). 


\section{DISCUSSION}

Evidence for commingling has been suggested by some as a necessary (though not sufficient) condition for rejection of the null hypothesis of no major gene effect [Morton et al., 1977]. To others, commingling is neither necessary nor sufficient [Murphy, 1964; Elston, 1979]. In the present study, data were simulated to satisfy the theoretical assumptions, on a population level, of a trait under the influence of a single locus with dominance together with additive polygenes and individual-specific environmental factors. The sampling design used has been previously shown to be efficient to detect a single-locus effect when it is present [Burns et al., 1984; Boehnke et al., 1988]. Our simulation results suggest that evidence for commingling is not a necessary condition for identification of the presence of a single locus. A few family studies of quantitative traits in the literature have also shown evidence for a single locus from segregation analysis, while failing to find evidence for commingling [Meyers et al., 1982; Dorus et al., 1983].

It is not surprising that skewness in the sample distribution was the important characteristic for finding evidence for commingling. Others have noted the relationship between skewness and evidence for commingled distributions [MacLean et al., 1976]. An unexpected finding was that under the conditions simulated here, exclusion of the probands typically resulted in a more positive measure of skewness than inclusion of the probands (see Table II). In contrast, Chakraborty and Hanis [1987] showed that for quantitative traits influenced solely by polygenes and individual-specific environmental factors, skewness was reduced to its expected value under a normal distribution when probands were excluded; the probands in their study were also selected from the upper $5 \%$ tail of the population distribution. The difference in the effect of eliminating the probands on the estimate of the measure of skewness under a model of multivariate normality [Chakraborty and Hanis, 1987] and under the mixed genetic models simulated here exemplifies the difficulties inherent in analyzing non-randomly sampled family data.

While segregation analysis can take into account the relatedness of the observations as well as the method of selecting pedigrees, commingling analysis cannot. However, both methods of analysis involve a null hypothesis with two restrictions on the parameters, namely a proportion set equal to zero and, in the case of dominant inheritance, two equal means. As a result of the two restrictions on the parameters, we have assumed that the corresponding likelihood ratio statistics are distributed approximately as chi squared on 2 degrees of freedom when the null hypotheses are true [Rao, 1973]. However, since the null hypothesis value for the proportion is on the boundary [Self and Liang, 1987], and either setting the proportion to zero or setting the means equal is sufficient to yield the null hypothesis, standard asymptotic theory cannot be invoked to guarantee the distribution of the test statistics. This concern is partially allayed by the fact that exactly the same sort of parameter restrictions are specified under the null hypothesis for both methods. In addition, even large changes in the critical values did not qualitatively affect our finding that a substantial difference in classification existed for the commingling analysis compared to the segregation analysis.

Several analytical techniques, in addition to commingling analysis, have been proposed to screen for the presence of a single locus with a large effect on a quantitative trait. In families, the within-sibship variance is expected to be higher in those 
families where there is segregation at a single locus with a large effect on the trait [Hewitt et al., 1979]. A single locus with a large effect is also suggested if there is a significant relationship between the within-sibship variance and within-sibship mean [Fain, 1978]. Exploratory data techniques allow a graphical representation of the data to be compared to what would be expected if a single locus with a large effect is present [Karlin et al., 1979; Carmelli et al., 1979; Kammerer et al., 1984]. All of these techniques require family data while commingling analysis can be applied to samples of unrelated observations as well as family data. While there are several examples of applications of the exploratory techniques in the literature, commingling analysis is the screening technique most often used.

Our finding that commingling analysis provides a high proportion of false negatives (no evidence for commingling when segregation analysis supports the presence of a single locus) suggests that commingling analysis is not a useful screening technique for identifying samples of pedigrees for segregation analysis. Evidence for commingling is not necessary to confirm the presence of a single-locus effect and, as has been suggested by Morton [1982], the critical evidence comes from the segregation analysis. Our finding that evidence of commingling depends on the distributional characteristics alone while evidence of major locus segregation depends on the distributional characteristics as well as the transmission of the rarer allele from parent to offspring is consistent with the lower power of commingling analysis compared to segregation analysis (43.2\% compared to $74.8 \%$ for Model I and $77.6 \%$ compared to $98.4 \%$ for Model II). It has been noted by others that the presence of commingling is not sufficient to identify a single-locus effect and that power transformation should be used to remove skewness while testing for evidence of a mixture of distributions [Murphy, 1964; MacLean et al., 1976; Elston, 1979]. While in our study we did not consider any power transformations, the proportion of samples not showing evidence for major locus segregation when commingling analysis did support the notion that the presence of a mixture of distributions was very low $(10 / 216=4.6 \%$ in Model I and $3 / 388=0.8 \%$ in Model II).

In contrast, commingling analysis can provide guidance in the choice of initial parameter estimates for segregation analysis. Despite the bias in the commingling analysis parameter estimates, all estimates with the possible exception of the allele frequency were in absolute terms not far from the true parameter values. Further, some upward bias in the commingling parameter estimates was predictable based on the nonrandom sampling through the upper tail of the trait distribution. In principle, this allows the possibility for downward adjustment of these commingling analysis estimates for use as initial parameter estimates in the segregation analysis. Choice of initial parameter estimates is seldom an exact science.

In conclusion, since realistic situations exist in which lack of evidence for commingling does not rule out the existence of a single locus with a major effect on the trait of interest, commingling analysis has limited usefulness as a screening technique for the presence of a single locus. In contrast, while evidence for commingling is not proof of a single genetic locus, it does suggest the possibility that a single locus having a major effect on the trait is involved. Furthermore, commingling analysis can provide guidance in the choice of initial parameter estimates for subsequent segregation analysis. Thus, commingling analysis can use- 
fully be performed prior to segregation analysis, but lack of evidence for a mixture of distributions in the commingling analysis should not preclude subsequent segregation analysis.

\section{ACKNOWLEDGMENTS}

The authors thank Drs. Sandra Hasstedt and Jean-Marc Lalouel for developing and making available the computer programs PAP and GEMINI, respectively. This research was supported by grants GM41440 (MB), HL39107 (PPM), HL30428 (PPM), HL24489 (PPM), and HL14320 (TLB) from the National Institutes of Health. Computing support was provided by the University of Michigan.

\section{REFERENCES}

Boehnke M, Lange K (1984): Ascertainment and goodness of fit of variance component models for pedigree data. In Rao DC, Elston RC, Kuller LH, Feinleib M, Carter C, Havlik R (eds): "Genetic Epidemiology of Coronary Heart Disease: Past, Present, and Future." New York: Alan R. Liss, Inc., pp $173-192$.

Boehnke M, Young MR, Moll PP (1988): Comparison of sequential and fixed-structure sampling of pedigrees in complex segregation analysis of a quantitative trait. Am J Hum Genet 43:336-343.

Boerwinkle E, Turner ST, Weinshilboum R, Johnson M, Richelson E, Sing CF (1986): Analysis of the distribution of erythrocyte sodium-lithium countertransport in a sample representative of the general population. Genet Epidemiol 3:365-378.

Bogardus C, Lillioja S, Nyomba BL, Freymond D, Zurol F, Swinburn B, Knowler W, Howard B, Bennett $P$ (1988): Evidence for a single gene, codominant mode of inheritance of insulin resistance in Pima Indians. Diabetes 1:37 (Suppl. 1):91A.

Bucher KD, Schrott HG, Clarke WR, Lauer RM (1982): The Muscatine Cholesterol Family Study: Distribution of cholesterol levels within families of probands with high, low and middle cholesterol levels. J Chronic Dis 35:385-400.

Burns TL (1982): “Sampling Considerations for the Determination of Genetic Transmission Mechanisms in Quantitative Traits." Ph.D. thesis, University of Michigan, Ann Arbor.

Burns TL, Moll PP, Schork MA (1984): Comparisons of different sampling designs for the determination of genetic transmission mechanisms in quantitative traits. Am J Hum Genet 36:1060-1074.

Carmelli D, Karlin S, Williams R (1979): A class of indices to assess major-gene versus polygenic inheritance of distributive variables. In Sing CF, Skolnick M (eds): "Genetic Analysis of Common Diseases: Applications to Predictive Factors in Coronary Disease." New York: Alan R. Liss, Inc., pp 259-270.

Chakraborty R, Hanis CL (1987): Nonrandom sampling in human genetics: Skewness and kurtosis. Genet Epidemiol 4:87-101.

Daniel C, Wood FS (1980): “Fitting Equations to Data." New York: John Wiley, p 29.

Day NE (1969): Estimating the components of a mixture of normal distributions. Biometrika 56:463-474.

Dorus E, Cox NJ, Gibbons RD, Shaughnessy R, Pandey GN, Cloninger CR (1983): Lithium ion transport and affective disorders within families of bipolar patients: Identification of a major gene locus. Arch Gen Psychiatry 40:545-552.

Elston RC (1979): Major locus analysis for quantitative traits. Am J Hum Genet 31:655-661 .

Elston RC, Namboodiri KK, Glueck CJ, Fallat R, Tsang R, Leuba V (1975): Study of the genetic transmission of hypercholesterolemia and hypertriglyceridemia in a 195 member kindred. Ann Hum Genet 39:67-87.

Elston RC, Stewart J (1971): A general model for the genetic analysis of pedigree data. Hum Hered $21: 523-542$.

Fain PR (1978): Characteristics of simple sibship variance tests for detection of major loci and application to height, weight and spatial performance. Ann Hum Genet 42:109-120.

Friedlander Y, Kark JD, Cohen T, Kaufmann NA, Eisenberg S, Stein Y (1984): Admixture analysis of plasma cholesterol levels in a Jewish population. Hum Hered 34:82-94. 
Hasstedt SJ (1982): A mixed-model likelihood approximation on large pedigrees. Comput Biomed Res 15:295-307.

Hasstedt SJ, Cartwright PE (1981): “PAP: Pedigree Analysis Package." Tech. Rep. 13, Department of Medical Biophysics and Computing, University of Utah, Salt Lake City.

Hewitt D, Jones GDL, Godin GJ, Wraight D, Breckenridge WC, Little JA, Steiner G, Mishkel MA (1979): Nature of the familial influence on plasma lipid levels. Atherosclerosis 32:381-396.

Hopper JL, Mathews JD (1982): Extensions to multivariate normal models for pedigree analysis. Ann Hum Genet 46:373-383.

Kammerer CM, MacCluer JW, Bridges JM (1984): An evaluation of three statistics of structured exploratory data analysis. Am J Hum Genet 36:187-196.

Karlin S, Carmelli D, Williams R (1979): Index measures for assessing the mode of inheritance of continuously distributed traits. I. Theory and justifications. Theor Popul Biol 16:81-106.

Lalouel JM (1979): "GEMINI: A Computer Program for Optimization of a Nonlinear Function." Tech. Rep. 14, Department of Medical Biophysics and Computing, University of Utah, Salt Lake City.

Laskarzewski PM, Rao DC, Glueck CJ (1984): The Cincinnati Lipid Research Clinic Family Study: Analysis of commingling and family resemblance for fasting blood glucose. Genet Epidemiol 1:341-355.

MacLean CJ, Morton NE, Elston RC, Yee S (1976): Skewness in commingled distributions. Biometrics 32:695-699.

McGue M, Laskarzewski P, Rao DC, Glueck CJ (1983): The Cincinnati Lipid Research Clinic Family Study: Commingling in the distributions of lipids and lipoprotein concentrations. Hum Hered 33:223-230.

McLachlan GH, Basford KE (1988): “Mixture Models. Inference and Applications to Clustering." New York: Marcel Dekker, Inc., pp 13-18.

Meyers DA, Bias WB, Marsh DG (1982): A genetic study of total IgE levels in the Amish. Hum Hered 32:14-23.

Moll PP, Michels VV, Weidman WH, Kottke BA (1989): Genetic determination of plasma apolipoprotein AI in a population-based sample. Am J Hum Genet 44:124-139.

Morton NE (1982): "Outline of Genetic Epidemiology." New York: S. Karger, pp 42-43.

Morton NE, MacLean CJ, Kagan A, Gulbrandsen GL, Rhoads GG, Yee S, Lew R (1977): Commingling in distributions of lipids and related vaariables. Am J Hum Genet 29:52-59.

Morton NE, MacLean CJ (1974): Analysis of family resemblance. III. Complex segregation of quantitative traits. Am J Hum Genet 26:489-503.

Murphy EA (1964): One cause? Many causes? The argument from the bimodal distribution. J Chronic Dis 17:301-324.

Ott J (1979): Detection of rare major genes in lipid levels. Hum Genet 51:79-91.

Olson JM, Boehnke M, Neiswanger K, Roche AF, Siervogel RM (1989): Alternative genetic models for the inheritance of the phenylthiocarbamide (PTC) taste deficiency. Genet Epidemiol 6:423-434.

Price RA, Cox NJ, Spielman RS, Van Loon JA, Maidak BL, Weinshilboum RM (1988): Inheritance of human platelet thermolabile sulfotransferase (TL PST) activity. Genet Epidemiol 5:1-16.

Price RA, Sorensen TI, Stunkard AJ (1989): Component distributions of body mass index defining moderate and extreme overweight in Danish men and women. Am J Epidemiol 130:193-201.

Rao CR (1973): “Linear Statistical Inference and Its Applications," 2nd ed. New York: John Wiley, pp 417-420.

Rice J, McGuffin P, Shaskan EG (1982): A commingling analysis of platelet monoamine oxidase activity. Psychiatr Res 7:325-335.

Rice J, McGuffin P, Goldin LR, Shaskan EG, Gershon ES (1984): Platelet monamine oxidase (MAO) activity: Evidence for a single major locus. Am J Hum Genet 36:36-43.

Richelson E, Snyder K, Carlson J, Johnson M, Turner ST, Lumry A, Boerwinkle E, Sing CF (1986): Lithium ion transport by erythrocytes of randomly selected blood bank donors and manic-depressive patients: Lack of association with affective illness. Am J Psychiatry 143:457-462.

Self SG, Liang KY (1987): Asymptotic properties of maximum likelihood estimators and likelihood ratio tests under nonstandard conditions. J Am Stat Assoc 82:605-610.

Sharma K, Byard PJ, Rao DC (1984): Commingling in the distributions of fat-related measures in Punjabi families. Hum Hered 34:278-284. 
Sing CF, Boerwinkle E, Moll PP, Templeton AR (1988): Characterization of genes affecting quantitative traits in humans. In Weir BS, Eisen EJ, Goodman MM, Namkoong G (eds): "Proceedings of the Second International Conference on Quantitative Genetics." Sunderland, MA: Sinauer, pp 250-269.

Turner ST, Weidman WH, Michels VV, Reed TJ, Ormson CL, Fuller T, Sing CF (1989): Distribution of sodium-lithium countertransport and blood pressure in causasians five to eighty-nine years of age. Hypertension 13:378-391.

Turner ST, Johnson M, Boerwinkle E, Richelson E, Taswell HF, Sing CF (1985): Sodium-lithium countertransport and blood pressure in healthy blood donors. Hypertension 7:955-962.

Young MR, Boehnke M, Moll PP (1988): Correcting for single ascertainment by truncation for a quantitative trait. Am J Hum Genet 43:705-708.

Edited by G.P. Vogler and D.C. Rao 